\title{
Prevalence of Contralateral Tumors in Patients (D) with Follicular Variant of Papillary Thyroid Cancer
}

Michael C Sullivan, MD, Paul H Graham, MD, Erik K Alexander, MD, Daniel T Ruan, MD, FACS, Matthew A Nehs, MD, Atul A Gawande, MD, MPH, FACS, Francis D Moore Jr, MD, FACS, Brooke E Howitt, MD, Kyle C Strickland, MD, PhD, Jeffrey F Krane, MD, PhD, Justine A Barletta, MD, Nancy L Cho, MD, FACS

BACKGROUND: Thyroid lobectomy alone is being performed increasingly for patients with encapsulated follicular variant of papillary thyroid carcinoma (fvPTC). However, the prevalence of contralateral disease in these patients is unknown. We investigated the presence of synchronous disease in fvPTC to improve decision making about the extent of surgical resection and need for surveillance.

STUDY DESIGN: We performed a retrospective review of patients who underwent thyroid surgery from October 2009 to February 2013 with a diagnosis of fvPTC as their primary lesion. We collected information on patient demographics, nodule size, multifocality, fine-needle aspiration results, lymphovascular invasion, extrathyroidal extension, and lymph node metastasis. Tumors were divided into noninvasive and invasive/infiltrative fvPTC categories. Characteristics of solitary and bilateral fvPTC were compared.

RESULTS: We identified 124 patients with final pathology demonstrating fvPTC. The most common fine-needle aspiration diagnosis was "suspicious for malignancy" $(\mathrm{n}=53)$. Sixty-five contralateral tumors were identified in 44 of 124 patients $(35.5 \%)$ and included fvPTC $(\mathrm{n}=40)$, classical PTC $(\mathrm{n}=22)$, tall cell PTC $(\mathrm{n}=2)$, and follicular carcinoma $(\mathrm{n}=1)$. Fifty contralateral tumors were 1 to $5 \mathrm{~mm}, 10$ measured 6 to $9 \mathrm{~mm}$, and 5 were $\geq 10 \mathrm{~mm}$. Contralateral disease correlated significantly with lymphovascular invasion $(\mathrm{p}=0.037)$ and larger primary lesions $(\mathrm{p}=0.020)$. There was no significant difference noted in extrathyroidal extension or lymph node metastasis. Both noninvasive and invasive/infiltrative fvPTC demonstrated similar rates of contralateral disease.

CONCLUSIONS: Bilateral disease is common in fvPTC, primarily in the form of papillary microcarcinomas. Future monitoring of the contralateral lobe should be discussed with fvPTC patients who do not undergo completion thyroidectomy. (J Am Coll Surg 2017;224:1021-1027. (C) 2016 by the American College of Surgeons. Published by Elsevier Inc. All rights reserved.)

Thyroid cancer incidence has risen in recent years, with approximately 63,000 new cases reported in the US in 2014. ${ }^{1-4}$ The majority of these malignancies are well-differentiated thyroid cancers, including papillary

\section{CME questions for this article available at} http://jacscme.facs.org

Disclosure Information: Authors have nothing to disclose. Timothy J Eberlein, Editor-in-Chief, has nothing to disclose.

Presented at the 97th Annual Meeting of the New England Surgical Society, Boston, MA, September 2016.

Received September 30, 2016; Revised December 9, 2016; Accepted December 9, 2016. thyroid carcinoma (PTC) and follicular thyroid carcinoma. After the classical type (cPTC), the follicular variant of papillary thyroid carcinoma (fvPTC) is the most common subtype, accounting for up to $41 \%$ of

From the Department of Surgery (Sullivan, Ruan, Nehs, Gawande, Moore, Cho), Department of Medicine, Thyroid Unit, Division of Endocrinology, Diabetes, and Hypertension (Alexander), and Department of Pathology (Howitt, Strickland, Krane, Barletta), Brigham and Women's Hospital, Boston, MA, and Department of Surgical Oncology, The University of Texas MD Anderson Cancer Center, Houston, TX (Graham).

Correspondence address: Nancy L Cho, MD, FACS, Department of Surgery, Brigham and Women's Hospital, 75 Francis St, Boston, MA 02115. email: nlcho@partners.org 


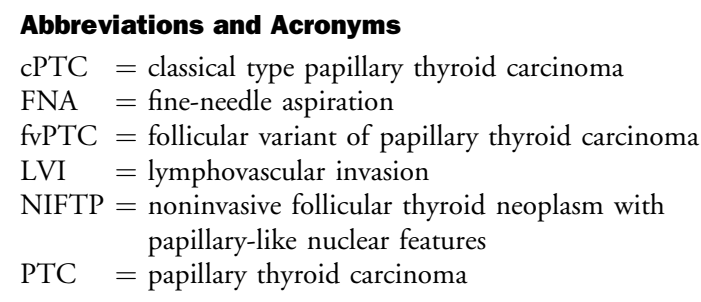

all PTC cases. ${ }^{5,6}$ As such, understanding the clinicopathologic behavior of fvPTC is critical for optimizing patient treatment plans.

Histologically, fvPTC is distinguished from cPTC by a follicular architecture with nuclear features typical of papillary carcinoma. Cytopathologic interpretation of fine-needle aspiration (FNA) samples for fvPTC can be difficult due to the fact that fvPTC often has more subtle nuclear features of PTC compared with cPTC, and FNA accuracy varies widely in the literature. ${ }^{7-9}$ The follicular variant of PTC also demonstrates considerable histopathologic overlap with benign lesions, such as follicular adenoma, confounding the diagnosis. Consequently, indeterminate cytology results are not uncommon, and these cases are often treated by diagnostic lobectomy for definitive diagnosis.

Substantial variability exists in the behavior of fvPTC histologic subtypes, depending on the growth pattern of the tumor. Encapsulated or noninvasive fvPTC possess a molecular profile similar to follicular adenomas/carcinomas, frequently displaying $R A S$ and lacking $B R A F V 600 \mathrm{E}$ mutations. ${ }^{10-14}$ These tumors are indolent compared with tumors with an infiltrative growth pattern, and have virtually no metastatic potential or recurrence risk in the absence of invasive growth. ${ }^{15-18}$ For example, Liu and colleagues ${ }^{18}$ found significantly greater rates of regional lymph node metastases among patients with infiltrative/diffuse neoplasms compared with their encapsulated counterparts ( $65 \%$ vs $5 \%$; $p<0.0001)$. Recently, Nikiforov and colleagues ${ }^{19}$ proposed a nomenclature revision for patients with noninvasive fvPTC, suggesting these cases should be termed noninvasive follicular thyroid neoplasm with papillary-like nuclear features (NIFTP) to reflect a more indolent phenotype and reduce overtreatment of these lesions.

The diagnosis of fvPTC on final pathology of lobectomy specimens leads to uncertainty about whether completion thyroidectomy is necessary, especially in the presence of untested contralateral nodules or potential microcarcinomas not yet seen on ultrasound. Patients with fvPTC have historically been treated similarly to those with cPTC, including thyroidectomy and radioactive iodine ablation, due in part to studies demonstrating equivalent prognosis., ${ }^{420-22}$ However, with the increased awareness about clinicopathologic differences in fvPTC subtypes, some authors have suggested that thyroid lobectomy alone might be appropriate for certain encapsulated tumors, such as NIFTP. ${ }^{17-19}$ In this study, we sought to define the prevalence of synchronous multifocal and bilateral disease in fvPTC to improve decision making about the extent of surgical resection.

\section{METHODS}

Under a protocol approved by the IRB at Brigham and Women's Hospital, we performed a retrospective review of all patients who underwent thyroid surgery at our institution between October 2009 and February 2013. For patients with a reported diagnosis of fvPTC for the index lesion, slides were re-reviewed by thyroid pathology subspecialists (JAB, BEH, and JFK). Tumors were categorized as fvPTC only if they had an entirely or almost entirely follicular architecture $(<1 \%$ papillary architecture) in combination with nuclear features of PTC. ${ }^{16}$ Cases were further divided into noninvasive and invasive/infiltrative fvPTC groups, as described previously. ${ }^{23}$ The noninvasive group would meet current diagnostic criteria of NIFTP, however, the diagnoses for all of these tumors were rendered before the proposed nomenclature revision.

Additional data, including patient demographics, FNA cytology results, extent of surgical resection; and histopathologic features, including nodule size, lymphovascular invasion (LVI), extrathyroidal extension, multifocality, and lymph node metastasis, were analyzed. Characteristics of fvPTC specimens with solitary vs bilateral disease were compared to define rates and features associated with bilateral disease. Solitary disease was defined as a single cancer identified in the entire thyroidectomy specimen; multifocal disease was defined as more than 1 cancer focus identified in either a single lobe or both thyroid lobes; and bilateral/ contralateral disease was defined as more than 1 cancer focus identified in both thyroid lobes.

The majority of FNA specimens were obtained at Brigham and Women's Hospital by an attending endocrinologist under ultrasound guidance in our Thyroid Nodule Clinic. Preoperative ultrasound was performed by an attending radiologist using a 5- to $17-\mathrm{MHz}$ transducer. Nodule size was reported in 3 dimensions (length, width, and depth). Ultrasound was used to guide the needle tip into the nodule and ensure an accurate specimen. Three (or rarely 4) needle sticks were obtained per nodule, constituting a single aspiration analysis. All aspirations were processed using the Thin Prep technique (Hologic, Inc). 
Each specimen was interpreted by a dedicated cytopathologist using The Bethesda System for Reporting Thyroid Cytopathology terminology. All outside cases were reviewed at our institution to confirm cytologic diagnosis and ensure there were no discrepancies in surgical management. Surgery was performed by 1 of 4 endocrine surgeons at the institution (FDM, AAG, DTR, and NLC). All specimens were submitted to pathology for permanent section, with stitches to mark the side and upper pole.

Data were analyzed using IBM SPSS Statistics, version 21.0 (IBM Corp). Significance was defined as $\mathrm{p} \leq 0.05$.

\section{RESULTS}

A total of 133 patients demonstrated fvPTC on final pathology from the index lesion. Nine patients underwent lobectomy alone and were excluded from the study due to the inability to analyze a complete thyroid specimen (Fig. 1). Of the remaining 124 patients, 85 patients underwent upfront total thyroidectomy and 39 underwent staged surgery (lobectomy followed by completion thyroidectomy). Mean $\pm \mathrm{SD}$ age was $50.6 \pm 13.0$ years. $\mathrm{Pa}$ tients were predominantly female (102 of 124 [82.3\%]). Mean \pm SD tumor size was $2.4 \pm 1.4 \mathrm{~cm}$ (range 0.1 to $6.4 \mathrm{~cm})$.

Analysis of preoperative cytology showed that the most common FNA diagnosis was "suspicious for malignancy" $(\mathrm{n}=53[42.7 \%])$, followed by "malignant" $(\mathrm{n}=20$ [16.1\%]), "atypia/follicular lesion of undetermined

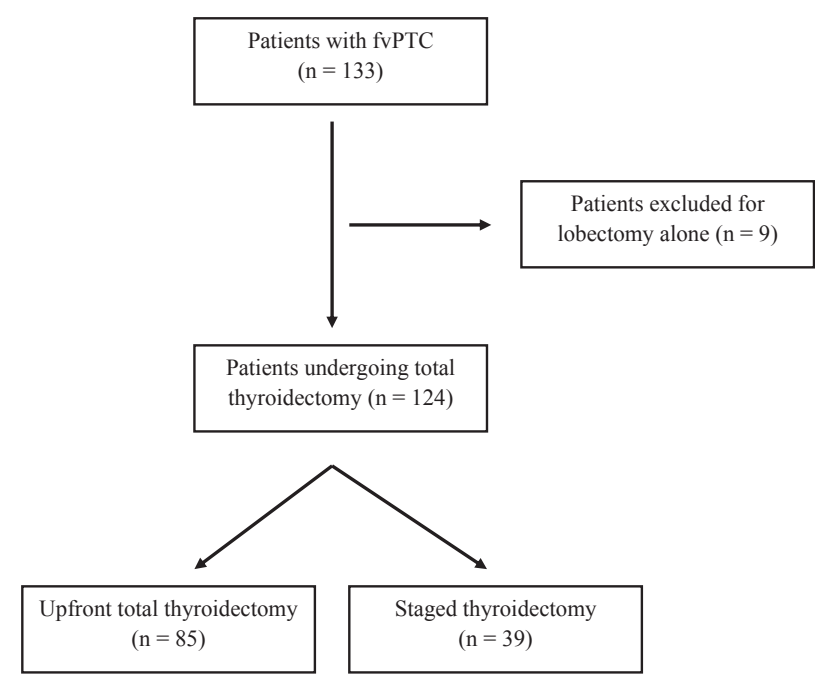

Figure 1. Study algorithm; 133 patients with follicular variant of papillary thyroid carcinoma (fVPTC) diagnosed on final pathology were identified during the study period. Of these, 85 patients underwent upfront total thyroidectomy and 39 underwent staged procedure (lobectomy followed by completion thyroidectomy). Nine patients underwent lobectomy only and were excluded from analysis. significance" ( $\mathrm{n}=19$ [15.3\%]), "suspicious for follicular neoplasm" ( $\mathrm{n}=9[7.3 \%])$, and "suspicious for Hurthle cell neoplasm" ( $\mathrm{n}=1[0.8 \%])$. Sixteen patients with preoperative benign FNA cytology underwent surgery for compressive symptoms or concern for malignancy due to large nodule size. Four samples (3.2\%) were nondiagnostic and 2 cases (1.6\%) did not have preoperative FNA performed (Table 1).

Based on histopathologic review of the entire thyroid specimen, we found that 58 cases $(46.8 \%)$ harbored solitary tumors and a high prevalence demonstrated multifocal disease $(n=66[53.2 \%])$ and contralateral tumors $(\mathrm{n}=44[35.5 \%])$. We counted a total of 65 tumors in the contralateral lobes of 44 patients represented by a variety of tumor types, most commonly fvPTC ( $\mathrm{n}=40 \mathrm{tu}-$ mors [61.5\%]) and cPTC $(\mathrm{n}=22$ tumors [33.8\%]) (Table 2). There were 2 cases of tall cell PTC and 1 case of follicular carcinoma identified in the contralateral lobe. One patient was noted to have $>10$ foci of papillary microcarcinomas (both follicular and classical types, ranging in size from 0.1 to $0.9 \mathrm{~cm}$ ) throughout the thyroid and was excluded from analysis due to the inability to precisely quantify these tumors. Mean tumor size in the contralateral lesions was $0.41 \mathrm{~cm}$ (range 0.1 to 3.5 $\mathrm{cm})$. Fifty contralateral tumors were between 1 and 5 $\mathrm{mm}, 10$ measured 6 to $9 \mathrm{~mm}$, and 5 were $\geq 10 \mathrm{~mm}$. Of the 5 contralateral tumors $\geq 10 \mathrm{~mm}$ in size (range 1.0 to $3.5 \mathrm{~cm}$ ), 4 were fvPTC and 1 was tall cell variant. Interestingly, all 5 of the primary fvPTC lesions in these patients were quite large (range 1.5 to $6.5 \mathrm{~cm}$ ). Three of these primary tumors were noninvasive fvPTC ranging from 3.0 to $4.3 \mathrm{~cm}$ in size. Additionally, the primary tumors frequently showed aggressive features, including 2 patients with LVI, 1 patient with extrathyroidal extension, and 2 patients with invasive fvPTC. Of note, we did not identify any noninvasive fvPTC cases with primary

Table 1. Fine-Needle Aspiration Results of 124 Follicular Variant of Papillary Thyroid Carcinoma Samples

\begin{tabular}{lcc}
\hline Fine-needle aspiration result & $\mathbf{n}$ & $\%$ \\
\hline Suspicious for malignancy & 53 & 42.7 \\
\hline Malignant & 20 & 16.1 \\
\hline $\begin{array}{l}\text { Atypia/follicular lesion of undetermined } \\
\text { significance }\end{array}$ & 19 & 15.3 \\
\hline Benign & 16 & 12.9 \\
\hline Suspicious for follicular neoplasm & 9 & 7.3 \\
\hline Suspicious for Hurthle cell neoplasm & 1 & 0.8 \\
\hline Nondiagnostic & 4 & 3.2 \\
\hline No fine-needle aspiration performed & 2 & 1.6 \\
\hline
\end{tabular}

Initial fine-needle aspiration results for patients with follicular variant of papillary thyroid carcinoma lesions showed that the majority of cytology results returned suspicious for malignancy $(\mathrm{n}=53[42.7 \%])$. 
Table 2. Histologic Variants of Contralateral Malignancies

\begin{tabular}{lcc}
\hline Pathology & $\mathbf{n}$ & $\%$ \\
\hline Follicular variant of papillary thyroid carcinoma & 40 & 61.5 \\
\hline Classical papillary thyroid carcinoma & 22 & 33.8 \\
\hline Tall cell papillary thyroid carcinoma & 2 & 3.8 \\
\hline Follicular cancer & 1 & 1.5 \\
\hline
\end{tabular}

Contralateral tumors were identified in 44 of 124 (35.5\%) patients. Follicular variant of papillary thyroid carcinoma was the most common histologic variant identified in the contralateral lobe.

tumors $<3.0 \mathrm{~cm}$ that demonstrated significant contralateral pathology $\geq 10 \mathrm{~mm}$ in size.

Finally, we analyzed patient and tumor characteristics among patients with solitary fvPTC and bilateral disease to determine factors associated with contralateral tumors. There was no significant between-group differences in age (solitary: $49.3 \pm 14.4$ years vs contralateral: $52.7 \pm 12.5$ years; $p=0.207$ ) or sex (solitary: 8 men vs contralateral: 12 men; $\mathrm{p}=0.089$ ) (Table 3 ). Tumor characteristics between the groups demonstrated that patients with contralateral disease had larger primary tumors (solitary: $2.2 \pm 1.2 \mathrm{~cm}$ vs contralateral: $2.8 \pm 1.5 \mathrm{~cm} ; \mathrm{p}=0.020)$. There were no significant differences in the frequency of extrathyroidal extension (solitary: 1 patient vs contralateral: 3 patients; $\mathrm{p}=0.313$ ) or presence of lymph node metastasis (solitary: 0 patients vs contralateral: 3 patients; $p=0.077$ ). Of note, LVI was significantly more common among patients with contralateral disease (solitary: 2 patients vs contralateral: 7 patients; $p=0.037$ ). Of the $7 \mathrm{pa}$ tients with both LVI and contralateral disease, 2 patients had contralateral fvPTC $>1 \mathrm{~cm}$ in size; the other 5 patients had contralateral microcarcinomas $<1 \mathrm{~cm}$ in size. When tumors were subdivided by noninvasive and invasive/infiltrative fvPTC subtypes, we identified $82(66.1 \%)$ noninvasive fvPTC neoplasms and $42(33.9 \%)$ invasive/infiltrative tumors. These cases were then divided into solitary and bilateral cancers. Both groups showed similar rates of contralateral malignancy (noninvasive: $40.3 \%$ vs invasive: $48.6 \%$; $\mathrm{p}=0.423)$.

\section{DISCUSSION}

The follicular variant of PTC is a unique entity with a range of biologic behaviors. Although the mainstay of treatment is surgical resection, there remains debate about the extent of surgery and its impact on recurrence and survival. As such, it is critical to delineate factors that can place patients at increased risk for compromised outcomes, including overly aggressive or unnecessary surgery. Here, we sought to identify factors that correlate with synchronous disease as the presence of multifocal or bilateral cancers can influence the degree of surgery or need for long-term surveillance. In our single-institution cohort, we found that $>53 \%$ of patients with fvPTC had multifocal disease, and $>35 \%$ had a contralateral malignancy. However, the majority of these lesions were incidental microcarcinomas. When compared with patients with solitary tumors, patients with contralateral disease had larger primary tumors and a greater frequency of LVI.

Previous studies have investigated the rate of incidental thyroid cancers after thyroidectomy for presumed benign disease. Campbell and colleagues ${ }^{24}$ showed that 31 of 134 patients $(23.1 \%)$ were found to have unsuspected thyroid cancer, including microcarcinomas, on final pathology after surgery for multinodular goiter. Similarly, Smith and colleagues $^{25}$ examined incidental cancer frequency in 2,551 patients after thyroidectomy for presumed benign disease (Graves' disease or nodular goiter) and found cancers in $238(15.6 \%)$ patients. In both series, cancer rates were higher than expected, although it is interesting to note that we found a considerably higher rate of incidental cancers in our fvPTC cohort $(53 \%$ multifocal and $35 \%$ contralateral). Two other studies identified comparably high rates (46.4\% [125 of 269 patients]

Table 3. Comparison of Follicular Variant of Papillary Thyroid Carcinoma Cases with Solitary vs Contralateral Disease

\begin{tabular}{lccc}
\hline Variable & Solitary $(\mathbf{n}=\mathbf{5 8})$ & Contralateral $(\mathbf{n}=\mathbf{4 4 )}$ & $\mathbf{p}$ Value \\
\hline Patient age, $\mathrm{y}$, mean \pm SD & $49.3 \pm 14.4$ & $52.7 \pm 12.5$ & 0.207 \\
\hline Male sex, $\mathrm{n}$ & 8 & 12 & 0.089 \\
\hline Primary size, cm, mean \pm SD & $2.2 \pm 1.2$ & $2.8 \pm 1.5$ & $0.020^{*}$ \\
\hline Extrathyroidal extension, n (\%) & $1(1.7)$ & $3(6.8)$ & 0.313 \\
\hline Lymph node metastasis, n (\%) & $0(0)$ & $3(6.8)$ & 0.077 \\
\hline Lymphovascular invasion, n (\%) & $2(3.4)$ & $7(15.9)$ & $0.037^{*}$ \\
\hline Noninvasive fvPTC, n (\%) & $40(59.7)$ & $27(40.3)$ & 0.423 \\
\hline Invasive/infiltrative fvPTC, n (\%) & $18(51.4)$ & $17(48.6)$ & 0.423 \\
\hline
\end{tabular}

Analysis of patient and tumor characteristics between solitary and bilateral fvPTC demonstrated that tumor size and lymphovascular invasion correlated significantly with contralateral disease. Both invasive/infiltrative and noninvasive tumors showed similar rates of contralateral malignancy.

*Significant.

fvPTC, follicular variant of papillary thyroid carcinoma. 
and $32 \%$ [ 426 of 1,313 patients]) of incidental contralateral malignancies in patients with well-differentiated papillary thyroid cancer, suggesting that patients with a suspected cancer diagnosis can harbor protumorigenic changes in the surrounding microenvironment that contribute to an overall predilection for forming additional cancers. ${ }^{26,27}$ Taken together, these data suggest that the rate of unsuspected incidental cancer is quite high, especially in the presence of a known cancer diagnosis. Consequently, patients who present with contralateral nodules $>1 \mathrm{~cm}$ during preoperative workup should undergo FNA sampling to rule out synchronous cancers before surgery.

Although contralateral disease was common in our series, these tumors were predominately microcarcinomas. The prognostic significance of residual microcarcinomas is not completely understood. Although earlier studies have raised concerns about the presence of residual thyroid leading to compromised long-term outcomes, ${ }^{28}$ recent investigations have called this idea into question. Adam and colleagues ${ }^{29}$ examining 61,775 patients with less-aggressive histologic variants of PTC, found that total thyroidectomy and lobectomy had similar overall survival rates. However, given the low rate of disease-specific mortality in patients with PTC, it remains unclear whether survival is an ideal metric of comparison between surgical strategies. Retrospectively analyzing 889 patients with pT1-2 well-differentiated thyroid cancer, Nixon and colleagues $^{30}$ found that patients undergoing thyroid lobectomy had no difference in local or regional recurrence. In contrast, Ross and colleagues ${ }^{31}$ showed that in 611 patients, micropapillary multifocal disease, in particular, demonstrated reduced risk of recurrence after total thyroidectomy $(6 \%)$ compared with patients who underwent less than complete resection (18\%). Given that the majority of our fvPTC cohort demonstrated multifocal disease, patients who undergo lobectomy alone might benefit from ongoing monitoring of the contralateral side, with the understanding that the likelihood of a clinically significant cancer developing is low.

In our population, we found that patients with larger primary tumors and those demonstrating LVI were considerably more likely to exhibit contralateral disease. Patients with LVI were nearly 5 times more likely to demonstrate contralateral disease than solitary disease. In addition, 2 of 5 cases with clinically significant disease $(>1 \mathrm{~cm})$ on the contralateral side showed LVI in the index lesion. Not surprisingly, LVI is associated with more aggressive disease in patients with PTC, including cervical lymph node involvement ${ }^{32}$ and distant metastases. ${ }^{33}$ In addition, LVI was the only significant prognostic indicator of recurrence in patients with papillary thyroid microcarcinoma after resection. ${ }^{34}$ According to the 2015 American Thyroid Association Guidelines, the presence of LVI indicates intermediate risk of recurrence with recommendation for total thyroidectomy, followed by postoperative radioactive iodine ablation. ${ }^{35}$ Consistent with this guideline, completion thyroidectomy should be discussed with patients when a diagnosis of fvPTC with LVI is obtained in thyroid lobectomy specimens.

Nikiforov and colleagues ${ }^{19}$ recently demonstrated that 67 of 109 patients with NIFTP treated with lobectomy alone were disease-free at follow-up (range 10 to 26 years). In contrast, $12 \%$ of patients with invasive fvPTC demonstrated adverse outcomes, including distant disease, death, local recurrence, and detectable serum thyroglobulin levels. Subsequently, the consensus group recommended against completion thyroidectomy and radioactive iodine ablation for NIFTP in an effort to avoid overtreatment. In our cohort, we identified $82(66.1 \%)$ cases that would meet current diagnostic criteria of NIFTP and 42 (33.9\%) invasive/infiltrative tumors. We found that a similar proportion of patients with noninvasive and invasive/infiltrative disease demonstrated contralateral disease. There was a trend toward increased contralateral disease in the invasive group relative to the noninvasive group ( $48.6 \%$ vs $40.3 \%$ ), but this finding was not significant. Given these data, lobectomy alone might be justified in the NIFTP population, although patients should be counseled about the potential for development of contralateral nodules and appropriate monitoring should be discussed accordingly.

The issue of overtreatment for indolent disease is not trivial, as completion surgery carries associated risks. In addition to the inherent difficulty of reoperative surgery, the contralateral parathyroids and recurrent nerve are placed at increased risk for injury during the procedure. In addition, patients now require lifelong thyroid hormone replacement after completion surgery, which can take time to titrate accurately. Despite this, completion thyroidectomy is generally well tolerated by most patients; 1 meta-analysis investigating 7 studies with 1,208 patients found no significant difference in complication rates (eg temporary or permanent recurrent laryngeal nerve injury, temporary or permanent hypocalcemia, hematoma, and wound infection) between completion thyroidectomy and upfront total thyroidectomy. ${ }^{36}$ As such, risks and benefits of completion surgery should be carefully discussed with patients and treatment plans reviewed in conjunction with endocrinologists and pathologists, as available.

Limitations of this investigation include its retrospective design and sample size. Larger, multi-institutional studies can be helpful to identify additional clinicopathologic or molecular parameters that could aid in 
management decisions. We did not characterize the contralateral fvPTC as noninvasive or invasive/infiltrative tumors, thereby limiting the conclusions about the clinical significance of these contralateral tumors. Additionally, although we believe our noninvasive group meets current diagnostic criteria of NIFTP, the diagnoses for these tumors were rendered before the formalized nomenclature revision; as such, we opted not to perform additional subanalyses on this tumor group. Finally, with no control group, it is impossible to postulate which of the observed contralateral malignancies would ultimately represent clinically significant disease. Despite these limitations, we believe our findings are novel and relevant to the fvPTC literature, especially given the recent paradigm shift toward de-escalating treatment of NIFTP cases.

\section{CONCLUSIONS}

Bilateral disease is common in fvPTC, primarily in the form of papillary microcarcinomas. Lymphovascular invasion and primary nodule size correlate significantly with the presence of contralateral disease, and noninvasive and invasive/infiltrative subtypes of fvPTC carry similar rates of contralateral tumors. Completion surgery should be discussed in fvPTC patients with LVI or invasive/infiltrative growth, in keeping with current American Thyroid Association guidelines. Patients who undergo lobectomy alone should be counseled on the potential for development of contralateral nodules and future surveillance monitoring should be discussed accordingly.

\section{Author Contributions}

Study conception and design: Cho

Acquisition of data: Sullivan, Graham, Alexander, Ruan, Nehs, Gawande, Moore, Howitt, Strickland, Krane, Barletta, Cho

Analysis and interpretation of data: Sullivan, Graham, Cho

Drafting of manuscript: Sullivan, Graham, Cho

Critical revision: Sullivan, Graham, Alexander, Ruan, Nehs, Gawande, Moore, Howitt, Strickland, Krane, Barletta, Cho

\section{REFERENCES}

1. Segel R, Ma J, Zou Z, et al. Cancer statistics, 2014. CA Cancer J Clin 2014;64:9-29.

2. Davies L, Welch HG. Increasing incidence of thyroid cancer in the United States, 1973-2002. JAMA 2006;295:2164-2167.

3. Morris LGT, Sikora AG, Tosteson TD, et al. The increasing incidence of thyroid cancer: the influence of access to care. Thyroid 2013;23:885-891.

4. Davies L, Morris LG, Haymart M, et al. American Association of Clinical Endocrinologists and American College of
Endocrinology disease state clinical review: the increasing incidence of thyroid cancer. Endocr Pract 2015;21:686-696.

5. Zidan J, Karen D, Stein M, et al. Pure versus follicular variant of papillary thyroid carcinoma: clinical features, prognostic factors, treatment and survival. Cancer 2003;97:1181-1185.

6. Finnerty BM, Kleiman DA, Scognamiglio T, et al. Navigating the management of follicular variant papillary thyroid carcinoma subtypes: a classic PTC comparison. Ann Surg Oncol 2015;22:1200-1206.

7. Ustun B, Chhieng D, Prasad ML, et al. Follicular variant of papillary thyroid carcinoma: accuracy of FNA diagnosis and implications for patient management. Endocr Pathol 2014;25:257-264.

8. Shih SR, Shun CT, Su DH, et al. Follicular variant of papillary thyroid carcinoma: diagnostic limitations of fine needle aspiration cytology. Acta Cytol 2005;49:383-386.

9. Kurian EM, Dawlett M, Wang J, et al. The triage efficacy of fine needle aspiration biopsy for follicular variant of papillary thyroid carcinoma using the Bethesda Reporting Guidelines. Diag Cytopathol 2011;10:E69-E73.

10. Xu B, Ghossein R. Encapsulated thyroid carcinoma of follicular cell origin. Endocr Pathol 2015;26:191-199.

11. Rivera M, Ricarte-Filho J, Knauf J, et al. Molecular genotyping of papillary thyroid carcinoma follicular variant according to its histological subtypes (encapsulated vs infiltrative) reveals distinct BRAF and RAS mutation patterns. Mod Pathol 2010; 23:1191-2000.

12. Mcfadden DG, Dias-Santagata D, Sado PM, et al. Identification of oncogenic mutations and gene fusions in the follicular variant of papillary thyroid carcinoma. J Clin Endocrinol Metab 2014;99:E2457-E2462.

13. Howitt BE, Jia Y, Sholl LM, et al. Molecular alteration in partially-encapsulated or well-circumscribed follicular variant of papillary thyroid carcinoma. Thyroid 2013;23:1256-1262.

14. Cancer Genome Atlas Research Network. Integrated genomic characterization of papillary thyroid carcinoma. Cell 2014; 159:676-690.

15. Gupta S, Ajise O, Dultz L, et al. Follicular variant of papillary thyroid cancer: encapsulated, nonencapsulated, and diffuse: distinct biologic and clinical entities. Arch Otolaryngol Head Neck Surg 2012;138:227-233.

16. Vivero M, Kraft S, Barletta JA. Risk stratification of follicular variant of papillary thyroid carcinoma. Thyroid 2013;23: $273-279$.

17. Rivera M, Tuttle RM, Patel S, et al. Encapsulated papillary thyroid carcinoma: a clinico-pathologic study of 106 cases with emphasis on its morphologic subtypes (histologic growth pattern). Thyroid 2009;19:119-127.

18. Liu J, Singh B, Tallini G, et al. Follicular variant of papillary thyroid carcinoma: a clinicopathologic study of a problematic entity. Cancer 2006;107:1255-1264.

19. Nikiforov YE, Seethala RR, Tallini G, et al. Nomenclature revision of encapsulated follicular variant of papillary thyroid carcinoma. A paradigm shift to reduce overtreatment of indolent tumors. JAMA Oncol 2016;2:1023-1029.

20. Passler C, Pragar G, Scheuba C, et al. Follicular variant of papillary thyroid carcinoma. Arch Surg 2003;138:1362-1366.

21. Lin HW, Bhattacharyya N. Clinical behavior of follicular variant of papillary thyroid carcinoma: presentation and survival. Laryngoscope 2010;120:712-716.

22. Yu XM, Schneider DF, Leverson G, et al. Follicular variant of papillary thyroid carcinoma is a unique clinic entity: a population-based study of 10,740 cases. Thyroid 2013;23:1263-1268. 
23. Strickland KC, Howitt BE, Marqusee E, et al. The impact of noninvasive follicular variant of papillary thyroid carcinoma on rates of malignancy for fine-needle aspiration diagnostic categories. Thyroid 2015;25:987-992.

24. Campbell MJ, Seib CD, Candell L, et al. The underestimated risk of cancer in patients with multinodular goiters after a benign fine needle aspiration. World J Surg 2015;39:695-700.

25. Smith JJ, Chen X, Schneider DF, et al. Cancer after thyroidectomy: a multi-institutional experience with 1,523 patients. J Am Coll Surg 2013;261:571-577.

26. Ricci JA, Alfonso AE. Multifocal micropapillary thyroid cancer: a new indication for total thyroidectomy? Am Surg 2012; 78:1211-1214.

27. Lodewijk L, Kluijfhout WP, Kist JW, et al. Characteristics of contralateral carcinomas in patients with differentiated thyroid cancer larger than $1 \mathrm{~cm}$. Langenbecks Arch Surg 2016;401: 365-373.

28. Bilimoria KY, Bentrem DJ, Ko CY, et al. Extent of surgery affects survival for papillary thyroid cancer. Ann Surg 2007;246: $375-381$.

29. Adam MA, Pura J, Gu L, et al. Extent of surgery for papillary thyroid cancer is not associated with survival: an analysis of 61,775 patients. Ann Surg 2014;260:601-605.

30. Nixon IJ, Ganly I, Patel SG, et al. Thyroid lobectomy for treatment of well differentiated intrathyroid malignancy. Surgery 2012;151:571-579.

31. Ross DS, Litofsky D, Ain KB, et al. Recurrence after treatment of micropapillary thyroid cancer. Thyroid 2009;19:1043-1048.

32. Kim JM, Kim TY, Kim WB, et al. Lymphovascular invasion is associated with lateral cervical lymph node metastasis in papillary thyroid carcinoma. Laryngoscope 2006;116:2081-2085.

33. Falvo L, Catania A, D’Andrea V, et al. Prognostic importance of histologic vascular invasion in papillary thyroid carcinoma. Ann Surg 2005;241:640-646.

34. Arora N, Turbendian HK, Kato MA, et al. Papillary thyroid carcinoma and microcarcinoma: is there a need to distinguish the two? Thyroid 2009;19:473-477.

35. Haugen BR, Alexander EK, Bible KC, et al. 2015 American thyroid association management guidelines for adult patients with thyroid nodules and differentiated thyroid cancer. Thyroid 2016;26:1-133.

36. Li YJ, Wang YZ, Yi ZB, et al. Comparison of completion thyroidectomy and primary total surgery for differentiated thyroid cancer: a meta-analysis. Oncol Res Treat 2015;38:528-531.

\section{Invited Commentary}

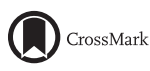

Jack M Monchik, MD, FACS

Providence, RI

This study was done before important molecular nomenclature changes, making it important to review these changes to appropriately provide commentary on this article.

A diagnosis of follicular variant of papillary carcinoma (FVPTC) presents multiple diagnostic and treatment challenges to the surgeon and pathologist. The recent American Thyroid Association (ATA) Guidelines advocate careful risk stratification and a measured approach to the diagnosis and treatment of thyroid cancer to minimize overtreatment. ${ }^{1}$ Patients with FVPTC have traditionally been treated as classical papillary thyroid cancer patients, with thyroidectomy and radioactive iodine ablation, based on previous studies showing equivalent prognosis. ${ }^{2}$ Two subsets of FVPTC have been identified: infiltrative (nonencapsulated) and encapsulated. $^{3}$ Encapsulated papillary carcinoma (EFVPTC) has dramatically increased in frequency over the past 2 to 3 decades and currently accounts for $10 \%$ to $20 \%$ of all thyroid cancers. ${ }^{4}$ The EFVPTC has been further subdivided into invasive and noninvasive categories to reflect the different biologic and clinical characteristics of these entities. The noninvasive component of EFVPTC is defined as having no evidence of vascular and/or tumor capsule invasion. An international, multidisciplinary retrospective study was performed of patients with noninvasive vs those with the invasive form of EFVPTC; it demonstrated no evidence of disease in a follow-up of 10 to 26 years in 109 patients with noninvasive EFVPTC, 67 of whom had only lobectomy, and no patient received radioactive iodine ablation. In the invasive group of EFVPTC patients, an adverse event was present in $12 \%$ of $101 \mathrm{pa}-$ tients. The adverse events included 5 patients with distant metastasis and 2 deaths. Based on this study and 352 additional well-documented cases in the literature with less than a $1 \%$ recurrence rate, the name noninvasive follicular thyroid neoplasm with papillary nuclear features (NIFTP) was adopted to reflect the indolent tumor behavior in this group of patients.

In the study by Sullivan and associates, their definition of noninvasive FVPTC is, for all practical purposes, the same as the definition of NIFTP. Their definition differs only in that they included some tumors with partial encapsulation. A high rate of a contralateral malignancy in patients with FVPTC was documented in this study. A contralateral malignancy was identified in 44 of the $124(35.4 \%)$ patients. Although 65 contralateral tumors were identified in these 44 patients, only 5 tumors were greater than $10 \mathrm{~mm}$. Preoperative fine-needle aspiration sampling may have identified these lesions. The most common tumor in the contralateral lobe was FVPTC. There was no significant difference in the incidence of contralateral tumors in the noninvasive (NIFTP) vs the invasive group. The primary tumors for each of these 5 patients were large, and in 3 of these 5 patients, the primary tumor was noninvasive (NIFTP) and ranged in size from 3 to $4.3 \mathrm{~cm}$. No primary tumor less than $3.0 \mathrm{~cm}$ had a contralateral tumor greater than $10 \mathrm{~mm}$. Contralateral disease correlated significantly 